\title{
Dental implants in a patient with a large idiopathic osteosclerosis of maxilla
}

\author{
Djanic Petar*, Brajdic Davor*, Biocic Josip*, Bajan Tomislav**, Salaric Ivan*, Peric \\ Berislav*, Macan Darko*
}

*University of Zagreb School of Dental Medicine, University Hospital Dubrava, Zagreb, Croatia

${ }^{* *}$ Private Dental Laboratory, Dugo Selo, Croatia

\section{Background:}

Numerous clinical studies have stated that the long-term survival of dental implants is more than $96 \%$. A 21 -year old healthy male patient referred at the Department of Oral and Maxillofacial surgery for a surgical removal of impact both upper right premolars before implant placement. The patient was followed from 2005 by his dentist and orthodontist who noticed that permanent dentition (teeth 13, 14, 15) had difficulties in erupting. This condition was caused by a large sclerotic, radiopaque bone abnormality that was asymptomatic and well delineated from surrounding normal bone.

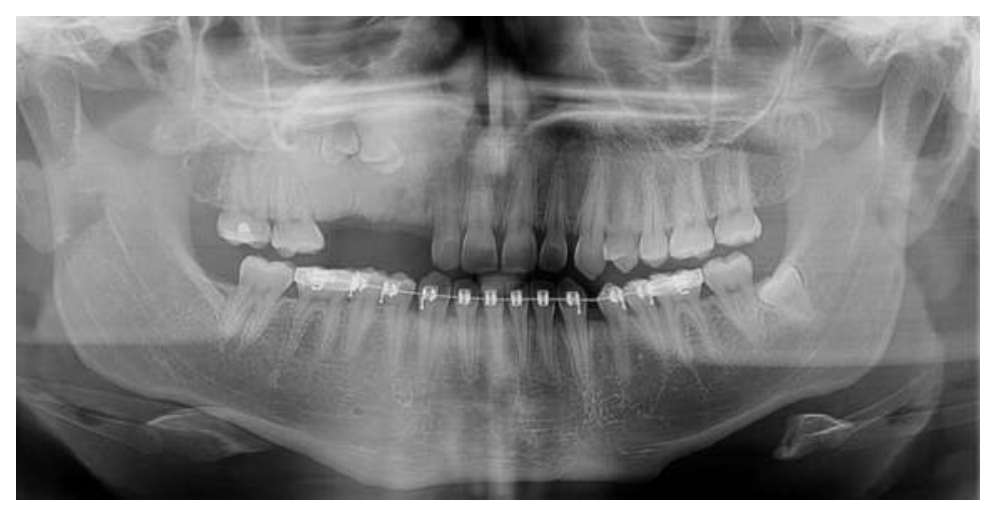

Picture 1. Preoperative panoramic x-ray.

\section{Aim/Hypothesis:}

There are different factors related to implant failures that can be divided in patient related factors, iatrogenic factors and dental implants factors. The aim of this presentation is to describe some important and simple steps that can improve early and long-term survival of dental implants in a patient with a large idiopathic osteosclerosis.
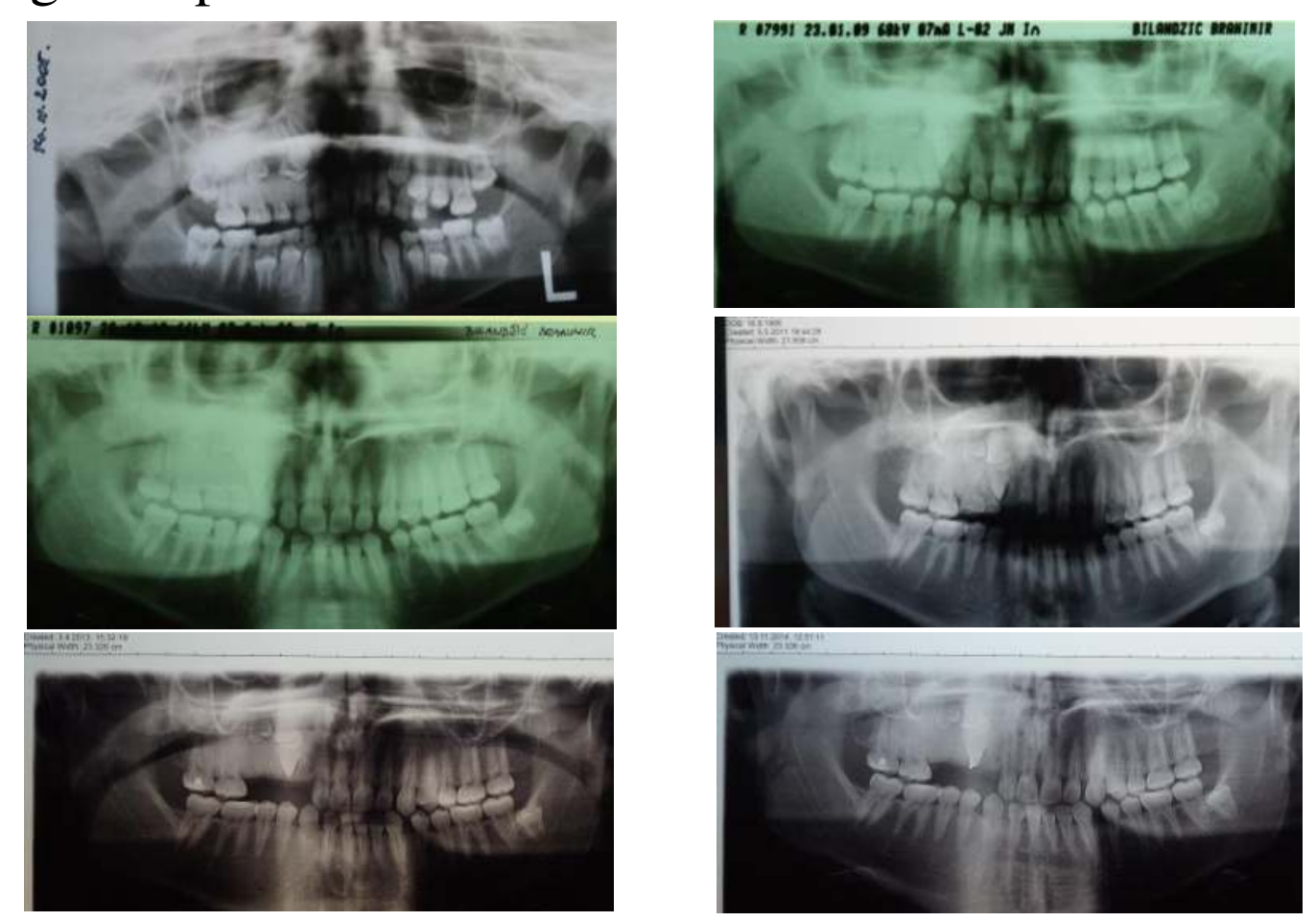

Pictures 2-7. Panoramic x-rays from 2005. till 2014. Osteosclerotic area of right maxiila with impacted upper right canine and both premolars.

Materials and Methods:

On initial clinical examination the patient presented with normal toothless canine and premolar regions. A CBCT analysis confirmed a large sclerotic area in right maxilla that impact the premolar teeth without displacement of adjacent teeth or bone expansion. In general anesthesia bone biopsy was taken and tooth 14 was removed. Tooth 15 was left because its removal would result in a large bone defect. The histopathology examination showed highly dens mature lamellar bone tissues. A diagnose of idiopathic osteosclerosis was made.
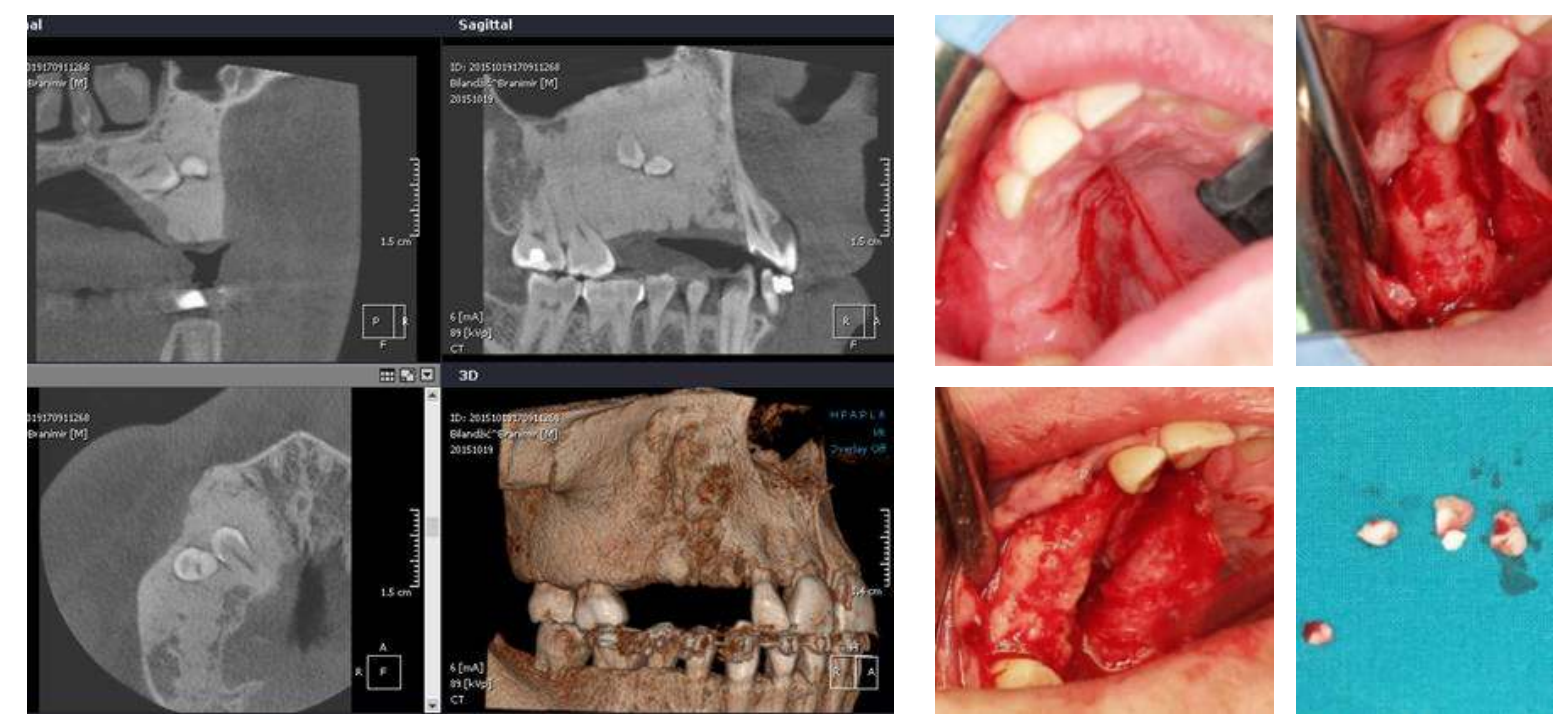

Picture 8. Preoperativ CBCT with impacted teeht 14,15. Picture 9. Intraoral preoperative view. Picture 10. Ridge after reflectin a flap. Picture 11. Palatal approach to alveolectomy of the tooth 14. Picture 12. Tooth 14.

After 2 months of uneventful healing 3 implants (Astra tech ${ }^{\circledR}$, Syrona Dentsplay, Germany) were inserted using the high dens bone protocol and the one-stage healing abutments were placed. The implants were chosen because of the good drilling protocol for dens bone and microthreads in the neck that can help to reduce bone stress and marginal bone loss. Also we used sterile saline that was cooled to $5^{\circ} \mathrm{C}$ for external irrigation to reduce heat generation and prevent osseous necrosis.
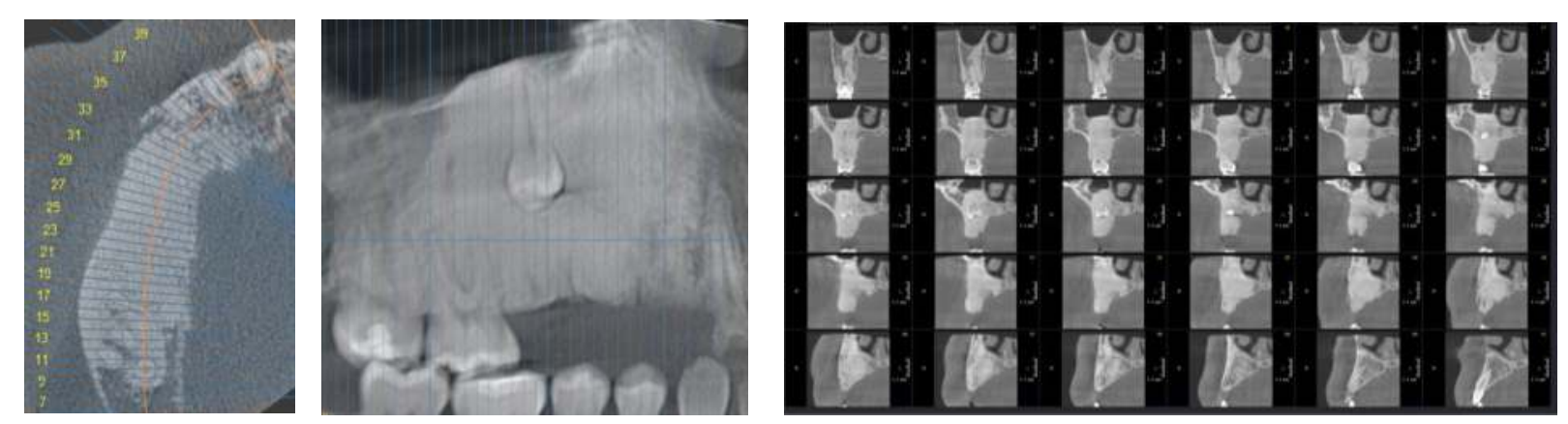

Pictures 13-15. CBCT before implant placement.

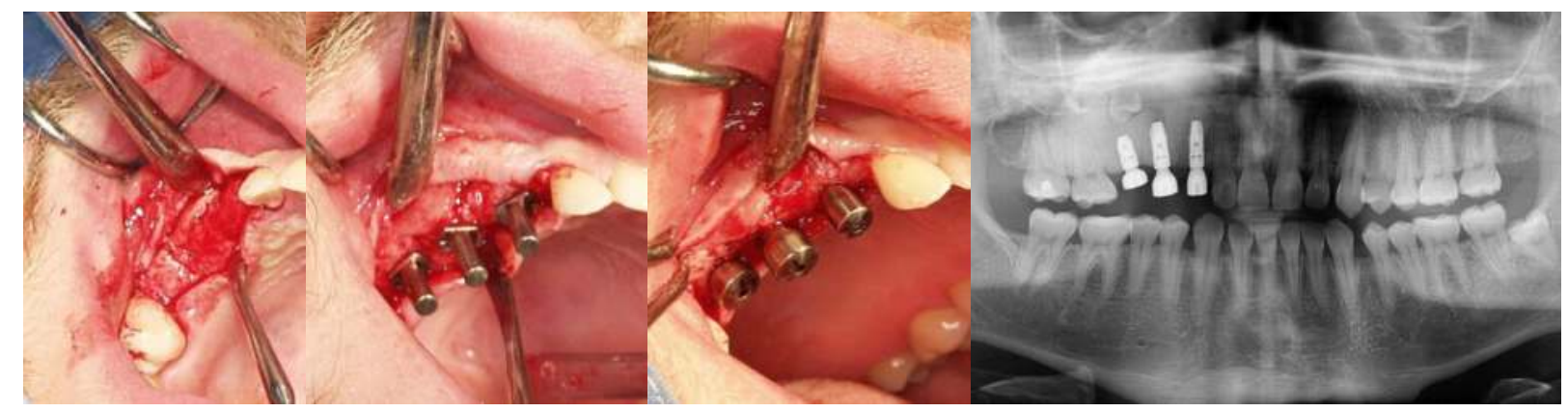

Pictures 16-18. Implant placement intraoral view. Picture 19. Panoramic x-ray six months after placement.

Six months after the uneventful healing period an individual screw-retained CAD-CAM zirconia oxide ceramic hybrid abutment-bridge was fabricated.
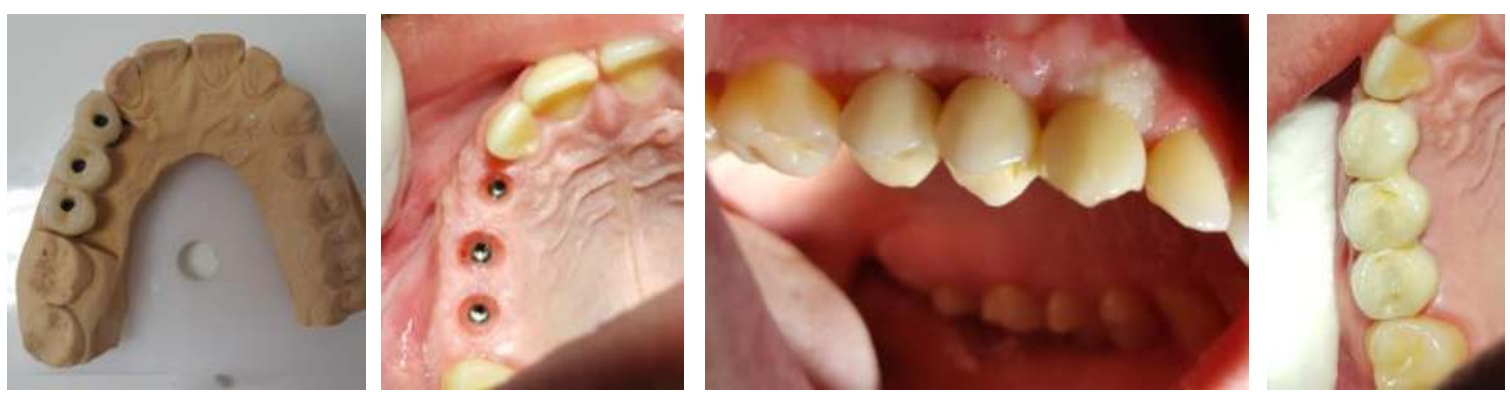

Picture 20. The bridge on model. Pictures 21-23. Intraoral view of screw-retained CAD-CAM zirconia oxide ceramic hybrid abutmentbridge.

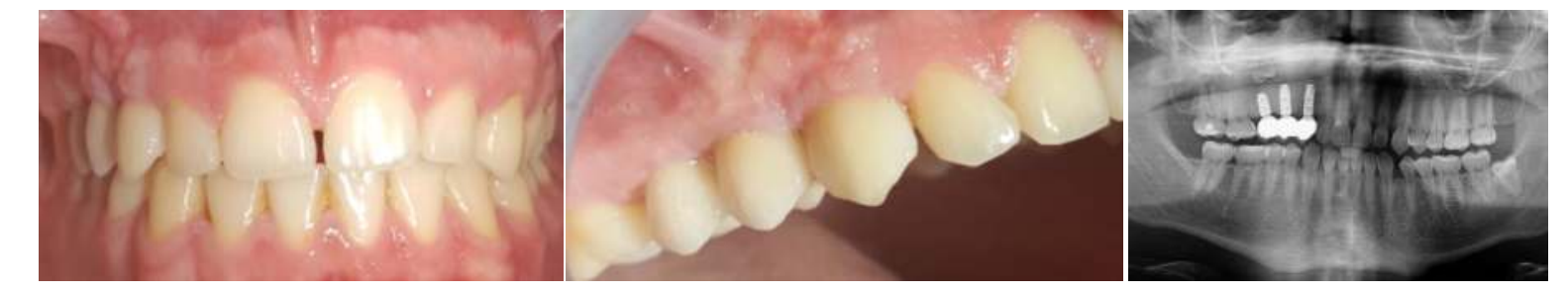

Pictures 24-26. Intraoral view 1 year follow up. Picture 27. Panoramic $\mathrm{x}$-ray 1 year follow up.

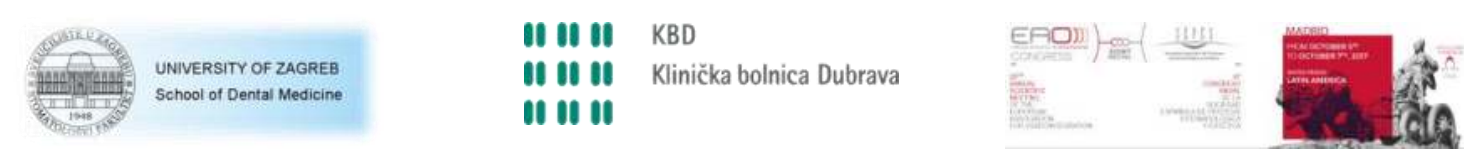

